\section{Central de regulação de leitos do SUS em Belo Horizonte, Minas Gerais, Brasil: avaliação de seu papel pelo estudo das internações por doenças isquêmicas do coração}

\author{
The hospital bed referral center under the Unified \\ National Health System in Belo Horizonte, Minas \\ Gerais State, Brazil: the case of hospitalization for \\ ischemic heart disease
}

Patrícia Alves Evangelista 1,2

Sandhi Maria Barreto 2,3

Henrique Leonardo Guerra 3

\footnotetext{
1 Secretaria Municipal de Saúde de Belo Horizonte, Belo Horizonte, Brasil. 2 Faculdade de Medicina, Universidade Federal de Minas Gerais, Belo Horizonte, Brasil.

3 Grupo de Pesquisa em Doenças Crônicas e Ocupacionais, Universidade Federal de Minas Gerais, Belo Horizonte, Brasil.

Correspondência P. A. Evangelista Secretaria Municipal de Saúde de Belo Horizonte. Rua Itapema 305, apto. 701, Belo Horizonte, $M G$ 30310-490, Brasil. patriciaa.e@terra.com.br pateva@pbh.gov.br
}

\begin{abstract}
The hospital admissions center in Belo Horizonte, Minas Gerais State, Brazil, aims to assure fast, timely, and equitable access to hospitalization services through the Unified National Health System. However, many patients are admitted directly to the hospitals, without going through the admissions center. This study compared the characteristics of hospitalizations in Belo Horizonte in 2002 according to type of access. All admissions for acute myocardial infarction and acute coronary disease were included. Of 3,705 admissions, $24.9 \%$ were processed through the hospital admissions center and 75.1\% through direct access. Direct hospitalizations were more common as compared to processing by the hospital admissions center for patients $\geq 70$ years ), those with presumptive diagnosis of acute coronary disease, to the surgical department, and on weekends. Admissions via the hospital admissions center were more common than direct access for patients residing outside Belo Horizonte, at non-public hospitals, and for the intensive care unit. Length-of-stay also varied according to type of access. The results confirm differences in the characteristics of admissions according to the two types of access.
\end{abstract}

Myocardial Ischemia; Health Services Accessibility; Hospitalization
Introdução

A superação das iniqüidades em saúde e em seus determinantes tem sido um grande desafio para diversos países no mundo. Políticas de saúde têm sido formuladas e implementadas tendo como princípio a eqüidade na distribuição de recursos, de bens e serviços essenciais à saúde. $\mathrm{O}$ Sistema Único de Saúde (SUS) no Brasil tem o compromisso de prestar assistência universal e integral, de forma equânime e de boa qualidade à população ${ }^{1}$. As demandas crescentes por uma assistência cada vez mais complexa, sem o correspondente crescimento da oferta de serviços, aumentam os desafios para que a eqüidade seja concretizada, com o acesso da população a uma assistência de forma adequada, oportuna e de acordo com suas necessidades.

Acesso ao cuidado de saúde é um conceito complexo, variando entre autores e em relação ao enfoque adotado, sendo geralmente empregado de forma imprecisa, mudando ao longo do tempo, e de acordo com o contexto ${ }^{2}$. Os conceitos de acesso e acessibilidade são mais abrangentes ou mais restritos, dependendo do autor. Acesso a serviços de saúde foi visto por Penchansky \& Thomas 3 como um conceito que resume um conjunto de dimensões específicas que descrevem a relação entre clientes e o sistema de cuidados de saúde: disponibilidade de serviços, acessibilidade aos serviços, acomodação entre forma de organização dos serviços e consideração desta, 
pelos clientes, como apropriada às suas necessidades, capacidade de compra dos serviços e aceitabilidade por ambas as partes. Frenk ${ }^{4}$ sugere um conceito de acessibilidade que reflete a relação complementar entre um conjunto de recursos da atenção à saúde e a população de usuários. Com relação a conceitos anteriormente elaborados, vê a acessibilidade como a relação funcional entre o conjunto de obstáculos (resistência) à busca $\mathrm{e}$ obtenção de atenção (distância, problemas de ordem financeira, formas de organização) e o correspondente poder de superação desses obstáculos por parte da população (poder de utilização). Donabedian 5 considera que a acessibilidade ao cuidado de saúde é um importante determinante da qualidade deste e o conceitua como a capacidade de obtenção de cuidado de forma fácil e conveniente, quando necessário.

Uma estratégia utilizada pelo SUS no Brasil para a garantia do acesso universal dos cidadãos aos serviços de saúde é a organização das centrais de regulação assistencial, por temas ou áreas assistenciais: obstétrica e neonatal, urgência e emergência, alta complexidade, transplantes, internações etc. Elas fazem o elo entre as demandas existentes e o recurso disponível, de modo a oferecer a melhor resposta assistencial, de forma equânime, de qualidade, e em tempo oportuno 6 .

Nessa perspectiva, a Secretaria Municipal de Saúde de Belo Horizonte (SMSA-BH) instituiu a Central de Internação como porta de entrada de seu sistema hospitalar, tendo a importante função de mediadora das internações, especialmente nos casos que requerem acesso mais rápido ao cuidado. Foi atribuído à Central de Internação o papel de controlar a capacidade instalada, os leitos disponíveis na rede assistencial, a ocupação de vagas e a viabilização do acesso aos procedimentos de média e alta complexidade e às internações. No fluxo proposto pela SMSA-BH, a Central de Internação deveria receber das unidades de atendimento todos os pedidos de internação e transferência de pacientes. Os médicos reguladores, por meio das informações clínicas fornecidas pelos serviços e profissionais das unidades de atendimento do paciente, classificam os pedidos de internação recebidos e disponibilizam os leitos disponíveis. Eles priorizam os casos de maior gravidade e complexidade, especialmente aqueles em que a demanda para internação é superior ao número de leitos disponíveis, como por exemplo, para a UTI, reduzindo o tempo de espera para obtenção do leito hospitalar. A Central de Internação, entretanto, não regula todas as internações hospitalares do SUS em Belo Horizonte (SUS-BH). No último quadrimestre de 2000, apenas $23 \%$ das internações foram encaminhadas pela Central de Internação; as restantes foram encaminhadas diretamente aos hospitais pelas unidades de urgência públicas ou contratadas no município ou pelos pronto-atendimentos hospitalares não credenciados ao SUS ( $A$ Central de Internação no Processo de Regulação do SUS-BH. Documento de circulação interna da SMSA-BH; 2001). Análise realizada em 2001 reforçou tais dados apontando que cerca de um terço das internações realizadas mensalmente pelo SUS-BH teve o pedido de vaga registrado na Central de Internação (Central de Internação. Documento de circulação interna da SMSA-BH; 2001). Contudo, não foram investigadas as discrepâncias existentes nas características das internações, entre as vias, nem os possíveis impactos nos desfechos das internações.

Estudos sobre acesso a serviços de saúde têm sido realizados no Brasil abordando a utilização de serviços ou acessibilidade particularmente nas suas dimensões geográfica, econômica, cultural e organizacional 7,8,9,10,11,12,13. Não estão disponíveis na literatura avaliações do desempenho das centrais de regulação de internações, sobretudo no que se relaciona à sua capacidade de agilizar o acesso aos serviços hospitalares, o que poderia fornecer subsídios para correções de desigualdades no uso dos serviços, de forma a se obter um sistema mais equânime.

O presente estudo pretende descrever e comparar, mediante análise dos dados do Sistema de Informações Hospitalares do SUS (SIH-SUS), as características das internações realizadas pelo SUS-BH em 2002 com relação à forma de acesso da população aos serviços hospitalares pela Central de Internação da SMSA-BH, ou de forma direta, sem ter a intermediação da Central. A escolha das internações por doenças isquêmicas do coração, como modelo, nesta avaliação, justifica-se por sua relevância na morbidade e mortalidade hospitalar no país e em Belo Horizonte, e pelo volume de internações hospitalares que produzem. Além disso, serão avaliadas as internações que tiveram suspeita inicial de infarto agudo do miocárdio e insuficiência coronariana aguda, pois são condições que freqüentemente levam pacientes à busca de cuidado de saúde de urgência. O fator tempo é importante para garantir a sobrevida desses pacientes, especialmente se diagnosticado um infarto agudo do miocárdio. Os serviços de atendimento às situações de urgência e emergência, ambulatoriais ou hospitalares, em Belo Horizonte, diante de pacientes com dor torácica aguda e com limitada disponibilidade de recursos diagnósticos e terapêuticos, necessitam transferir rapidamente seus pacientes para um hospital com melhores recursos para realização de propedêutica para 
confirmação diagnóstica e instituição de oportuno e adequado tratamento. Pela seleção destas doenças é possível, portanto, avaliar a capacidade reguladora da Central de Internação.

\section{Material e métodos}

\section{Fontes de dados}

Foram utilizados os bancos de dados contendo informações sobre solicitações de vaga à Central de Internação e internações realizadas pelo SUS, gerados e/ou consolidados na SMSA-BH no ano de 2002, armazenados em diferentes arquivos:

- Banco de dados das Autorizações de Internação Hospitalar (AIH) do Departamento de Informação e Informática do SUS/Ministério da Saúde (DATASUS) recebidas de todos os hospitais, digitadas por eles, e consolidados na SMSA-BH;

- Banco de dados dos laudos médicos para emissão de $\mathrm{AIH}$, digitados pela SMSA-BH (PRODABEL - Empresa de Informática e Informação do Município de Belo Horizonte);

- Banco de dados do registro de pedidos de vagas à Central de Internação, elaborado pela PRODABEL.

A integração dos arquivos ocorreu por intermédio da variável número da AIH e do número do laudo médico, com a criação de um novo arquivo para o estudo. O número do laudo médico é emitido pela Central de Internação no momento da confirmação da internação e acompanha todo o processo até o faturamento. As informações sobre solicitações de vaga para internações feitas à Central de Internação foram verificadas entre 1o de dezembro de 2001 até 31 de dezembro de 2002. A internação foi considerada mediada pela Central de Internação diante da presença das variáveis do registro de pedidos de vaga em data anterior ou no mesmo dia da data da internação. Caso contrário, ela foi considerada efetuada pelo fluxo direto com os hospitais, que será denominada daqui por diante como via direta.

\section{Fluxo de internações pelo SUS-BH}

A Figura 1 apresenta os possíveis fluxos percorridos para a internação dos pacientes pelo SUS-BH.

\section{- Fluxo mediado pela Central de Internação}

Diante da formulação de hipótese diagnóstica inicial e da avaliação da necessidade da internação de determinado paciente, o serviço de saúde que o atendeu aciona a Central de Internação da SMSA-BH por telefone, solicitando uma vaga para internação hospitalar e fornecendo o código de procedimento do SUS. Após o fornecimento dos dados de identificação e alguns sintomas e dados clínicos descrevendo a situação em que se encontra o paciente no momento, os teledigifonistas da Central de Internação buscam a vaga para o paciente no hospital municipal próprio ou nos serviços conveniados ao SUS. Duas situações podem ocorrer:

(i) A vaga é encontrada e o paciente será internado. A Central de Internação comunica ao serviço de origem o nome do hospital onde o paciente será internado e fornece ao hospital o número do laudo médico a ser preenchido;

(ii) $\mathrm{O}$ pedido de vaga é cancelado pelo serviço de origem por diversos motivos, sendo os principais: internação conseguida de forma direta com o hospital, internação no próprio serviço (caso o solicitante seja o ambulatório de um hospital e tenha havido liberação de um leito próprio), internação realizada de forma particular em leito não conveniado ao SUS, alteração do quadro clínico do paciente, óbito, mudança de diagnóstico e/ou mudança de tratamento, ou a situação deixou de ser uma urgência.

\section{- Fluxo da internação efetuada de forma direta}

Algumas vezes, as unidades solicitantes negociam a vaga para internação diretamente com o hospital, sem que haja pedido de vaga hospitalar para a Central de Internação. Somente após a internação do paciente é que o hospital solicita o número do laudo médico de internação à Central de Internação da SMSA-BH, que assim toma conhecimento da ocupação do leito hospitalar.

\section{População e período do estudo}

Foram estudadas as internações hospitalares realizadas em caráter de urgência e emergência pelo SUS-BH, entre 1o de dezembro de 2002 e 31 de dezembro de 2002, de pacientes de ambos os sexos, com 30 anos e mais de idade, independente do município de procedência. A solicitação inicial da internação pelo SUS é feita pelos procedimentos solicitados. Os procedimentos solicitados são aqueles que os profissionais de saúde colocam nos laudos médicos para justificar o pedido de internação, diante da hipótese diagnóstica inicial que poderá ser confirmada ou não durante a internação. Os seguintes procedimentos solicitados foram selecionados para o estudo porque são condições que freqüentemente levam os pacientes à busca de atendimento 

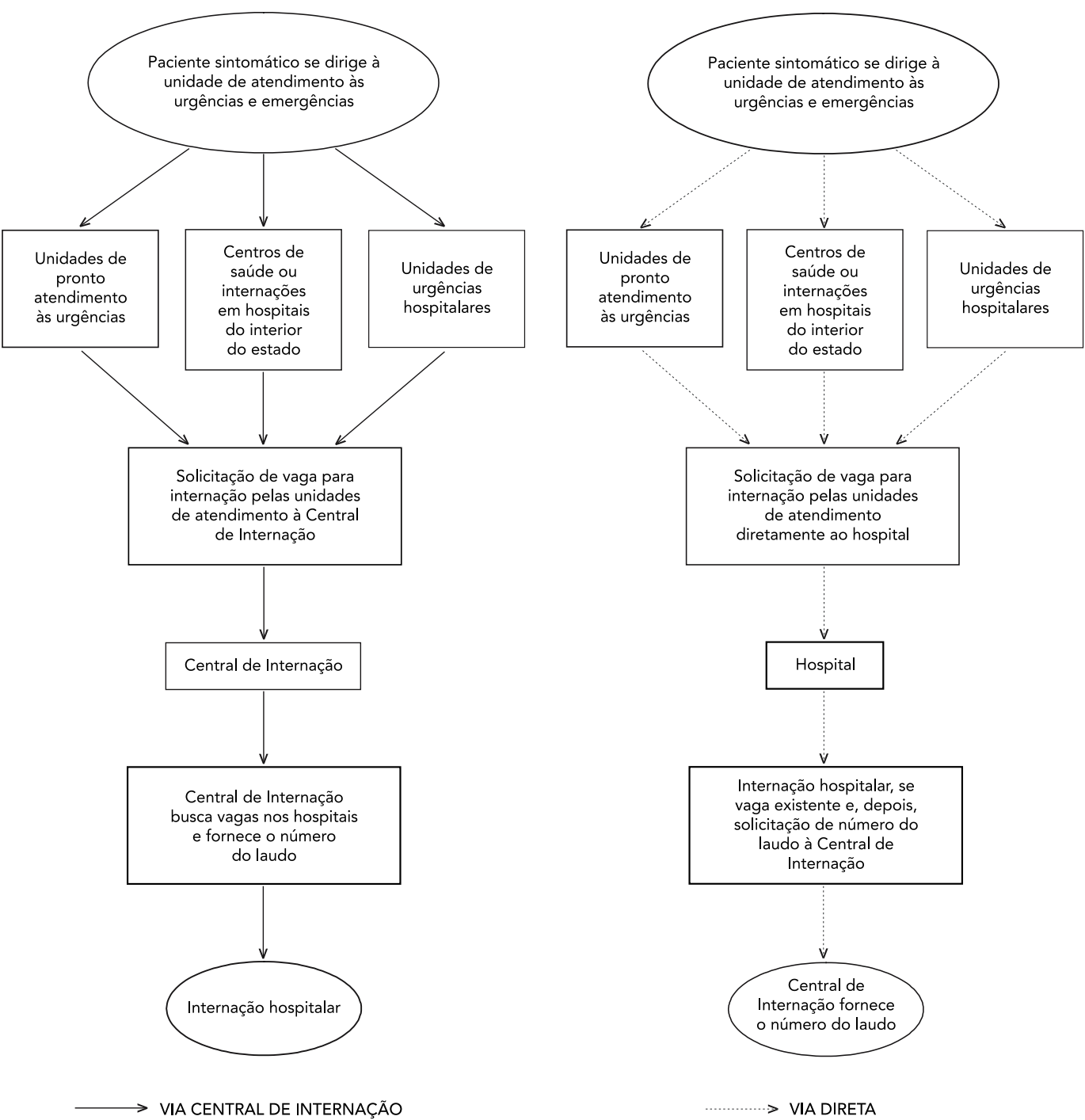

médico e à necessidade de hospitalização para realização de propedêutica para confirmação diagnóstica e/ou tratamento: infarto agudo do miocárdio (77.500.024) e insuficiência coronariana aguda (77.500.032). Tais procedimentos serão denominados daqui para frente "hipótese diagnóstica”. Mediante confirmação diagnóstica e tratamento realizado, os profissionais de saúde colocam nas AIH/SUS os procedimentos realizados e o diagnóstico principal pela Classificação Estatística Internacional de Doenças e Problemas Relacionados à Saúde, 10a Revisão (CID-10) 14.

\section{Análise dos dados}

Foram estudadas as seguintes variáveis: sexo (masculino e feminino); idade (intervalos de dez anos); hipótese diagnóstica (infarto agudo do miocárdio e insuficiência coronariana aguda); especialidade da internação (clínica cirúrgica e clínica médica); residência do paciente (Belo Horizonte e outros municípios); dia da internação (fim de semana, quando a internação ocorreu sábado e domingo e dia de semana, quando ocorreu de segunda a sexta); hospitais 
(públicos, agrupando nesta categoria os hospitais da esfera administrativa federal, municipal ou estadual e não públicos, se hospitais filantrópicos ou privados contratados ou conveniados ao SUS-BH); utilização de UTI durante a internação (sim e não). O teste do qui-quadrado de Pearson foi utilizado para avaliar as diferenças de proporções das variáveis categóricas entre as vias de internação. Foi realizada análise das medidas de dispersão das variáveis contínuas. Para comparação das médias de idade entre as vias de internação foi utilizada a análise de variância. As demais variáveis não apresentaram normalidade em sua distribuição mesmo após transformação logarítmica. Foi utilizado na análise, por conseguinte, um teste não paramétrico, o teste U de Mann-Whitney, que se baseia na distribuição e comparação das variáveis por postos. Os programas Epi Info 6.02d (Centers for Disease Control and Prevention, Atlanta, Estados Unidos) e SPSS 12.0 para Windows (SPSS Inc., Chicago, Estados Unidos) foram utilizados na análise.

\section{Aspectos éticos}

O estudo foi aprovado pela Secretaria Municipal de Saúde de Belo Horizonte e pelo Comitê de Ética em Pesquisa da Universidade Federal de Minas Gerais (parecer nº. ETIC 323/04).

\section{Resultados}

Foram realizadas 3.705 internações no período estudado, sendo $922(24,9 \%)$ delas pela Central de Internação e $2.783(75,1 \%)$ por via direta.

A Tabela 1 descreve as características das internações realizadas com relação à via de internação. Observou-se predomínio das internações

Características das internações hospitalares realizadas pelo Sistema Único de Saúde em Belo Horizonte, Minas Gerais, Brasil, segundo via de internação, 2002.

\begin{tabular}{|c|c|c|c|c|c|c|c|c|}
\hline \multirow[t]{2}{*}{ Variável } & \multicolumn{2}{|c|}{ Central de Internação } & \multicolumn{2}{|c|}{ Direta } & \multicolumn{2}{|c|}{ Total } & \multirow[t]{2}{*}{$\chi^{2}$} & \multirow[t]{2}{*}{$\mathbf{p}$} \\
\hline & $\mathbf{n}$ & $\%$ & $\mathrm{n}$ & $\%$ & $\mathbf{n}$ & $\%$ & & \\
\hline \multicolumn{9}{|l|}{ Sexo } \\
\hline Masculino & 533 & 57,8 & 1.517 & 54,5 & 2.050 & 55,3 & 3,05 & 0,081 \\
\hline Feminino & 389 & 42,2 & 1.266 & 45,5 & 1.655 & 44,7 & & \\
\hline \multicolumn{9}{|l|}{ Idade (anos) * } \\
\hline $30-39$ & 56 & 6,1 & 126 & 4,5 & 182 & 4,9 & 19,91 & 0,001 \\
\hline $40-49$ & 196 & 21,3 & 527 & 19,0 & 723 & 19,5 & & \\
\hline $50-59$ & 227 & 24,6 & 774 & 27,8 & 1.001 & 27,0 & & \\
\hline $60-69$ & 270 & 29,3 & 697 & 25,1 & 967 & 26,1 & & \\
\hline $70+$ & 173 & 18,8 & 657 & 23,6 & 830 & 22,4 & & \\
\hline \multicolumn{9}{|l|}{ Hipótese diagnóstica } \\
\hline Infarto agudo do miocárdio & 319 & 34,6 & 713 & 25,6 & 1.302 & 27,9 & 27,78 & $<0,001$ \\
\hline Insuficiência coronariana aguda & 603 & 65,4 & 2.070 & 74,4 & 2.673 & 72,1 & & \\
\hline \multicolumn{9}{|l|}{ Especialidade } \\
\hline Clínica cirúrgica & 50 & 5,4 & 254 & 9,1 & 304 & 8,2 & 12,61 & $<0,001$ \\
\hline Clínica médica & 872 & 94,6 & 2.529 & 90,9 & 3.401 & 91,8 & & \\
\hline \multicolumn{9}{|l|}{ Residência } \\
\hline Belo Horizonte & 518 & 56,2 & 1.787 & 64,2 & 2.305 & 62,2 & & \\
\hline Outros municípios & 404 & 43,8 & 996 & 35,8 & 1.400 & 37,8 & 18,99 & $<0,001$ \\
\hline \multicolumn{9}{|l|}{ Fim de semana } \\
\hline Sim & 162 & 17,6 & 581 & 20,9 & 743 & 20,1 & 4,72 & 0,030 \\
\hline Não & 760 & 82,4 & 2.202 & 79,1 & 2.962 & 79,9 & & \\
\hline \multicolumn{9}{|l|}{ Hospital público } \\
\hline Sim & 27 & 2,9 & 779 & 28,0 & 806 & 21,8 & 255,57 & $<0,001$ \\
\hline Não & 895 & 97,1 & 2.004 & 72,0 & 2.899 & 78,2 & & \\
\hline \multicolumn{9}{|l|}{ Utilização de UTI } \\
\hline Sim & 186 & 20,2 & 468 & 16,8 & 654 & 17,7 & 5,37 & 0,020 \\
\hline Não & 736 & 79,8 & 2.315 & 83,2 & 3.051 & 82,3 & & \\
\hline
\end{tabular}

* Duas idades ignoradas via direta. 
que corresponderam ao sexo masculino, não havendo diferenças significativas entre as vias de internação. A média de idade dos pacientes internados pela Central de Internação foi $58,3 \pm 12,1$ anos e 59,6 $\pm 12,5$ anos pela via direta. A proporção de pacientes com 70 anos e mais foi maior entre as internações por via direta do que pela Central de Internação (23,6\% e 18,8\%, respectivamente). A hipótese diagnóstica formulada com maior freqüência para justificar a internação foi insuficiência coronariana aguda nos dois grupos. Por via direta foram internados $74,4 \%$ de casos suspeitos de insuficiência coronariana aguda, sendo este percentual de 65,4 pela Central de Internação. A maioria das internações foi efetuada para tratamento clínico em leitos da especialidade clínica médica. Houve um maior percentual de internações para tratamento pela clínica cirúrgica pela via direta $(9,1 \%)$ que pela Central de Internação $(5,4 \%)$. Houve maior proporção de internações de pacientes que residiam em Belo Horizonte (62,2\%). A Central de Internação internou maior percentual de pacientes residentes em outros municípios $(43,8 \%)$ que a via direta $(35,8 \%)$. Verificou-se maior percentual de internações no fim de semana pela via direta $(20,9)$ em comparação à Central de Internação $(17,6)$. A maioria das internações foi realizada em hospitais não públicos $(78,2 \%)$. O percentual de internações nos hospitais públicos foi de 97,1 pela Central de Internação e 72 por via direta. A proporção de internações em que houve utilização de UTI foi maior pela Central de Internação $(20,2 \%)$ que por via direta $(16,8 \%)$.

A Tabela 2 apresenta a característica das internações segundo residência do paciente e hipótese diagnóstica. Entre os residentes em outros municípios, a proporção de internações por infarto agudo do miocárdio foi maior nas internações realizadas pela Central de Internação $(41,1 \%)$ do que nas internações realizadas por via direta (23,6\%). Não houve diferença estatistica- mente significativa entre hipótese diagnóstica e via, nas internações de residentes em Belo Horizonte.

A Tabela 3 apresenta a caracterização de variáveis selecionadas das internações. Nas internações com hipótese diagnóstica de infarto agudo do miocárdio, em que houve utilização de UTI, o número de dias utilizados foi diferente entre as vias. Verificou-se variação de 1 a 52 dias de uso de UTI (mediana = 3,0 dias) pela Central de Internação e variação de 1 a 25 dias (mediana = 3,0 dias) por via direta. Não houve diferença estatisticamente significativa, quando a hipótese diagnóstica à internação foi insuficiência coronariana aguda. O número de dias de internação variou de 1 a 44 nas internações realizadas pela Central de Internação (mediana $=6,0$ dias) e de 1 a 87 (mediana $=5,0$ dias) por via direta, quando a hipótese diagnóstica foi insuficiência coronariana aguda. Não houve diferenças estatisticamente significativas, quando a hipótese diagnóstica foi infarto agudo do miocárdio.

\section{Discussão}

Com base nos resultados encontrados, pode-se concluir que a Central de Internação não desempenhou plenamente o seu papel de instrumento regulador do acesso aos leitos hospitalares públicos e contratados, por essas hipóteses diagnósticas. O estudo confirmou, portanto, os dados das análises anteriormente realizadas pela SMSA-BH (A Central de Internação no Processo de Regulação do SUS-BH; 2001. Central de Internação; 2001. Documentos de circulação interna da SMSA-BH). Além disso, mostrou diferenças nas características das internações hospitalares segundo a forma de acesso às internações.

Um importante determinante do acesso e utilização de serviços, possível razão para a pequena proporção de casos em que a Central de

Internações realizadas pelo Sistema Único de Saúde em Belo Horizonte, Minas Gerais, Brasil, segundo residência dos pacientes, segundo hipótese diagnóstica e via de internação, 2002.

\begin{tabular}{|c|c|c|c|c|c|c|c|c|c|c|c|c|}
\hline \multirow[t]{3}{*}{ Hipótese diagnóstica } & \multicolumn{6}{|c|}{ Belo Horizonte } & \multicolumn{6}{|c|}{ Outros municípios } \\
\hline & \multicolumn{2}{|c|}{ Central de Internação } & \multicolumn{2}{|c|}{ Direta } & \multirow[t]{2}{*}{$\chi^{2}$} & \multirow[t]{2}{*}{$p$} & \multicolumn{2}{|c|}{ Central de Internação } & \multicolumn{2}{|c|}{ Direta } & \multirow[t]{2}{*}{$\chi^{2}$} & \multirow[t]{2}{*}{$p$} \\
\hline & $\mathrm{n}$ & $\%$ & $\mathrm{n}$ & $\%$ & & & $\mathrm{n}$ & $\%$ & $\mathrm{n}$ & $\%$ & & \\
\hline Infarto agudo miocárdio & 153 & 29,5 & 478 & 26,7 & 1,57 & 0,210 & 166 & 41,1 & 235 & 23,6 & 43,04 & $<0,001$ \\
\hline Insuficiência coronariana aguda & 365 & 70,5 & 1.309 & 73,3 & & & 238 & 58,9 & 761 & 76,4 & & \\
\hline Total & 518 & 100,0 & 1.787 & 100,0 & & & 404 & 100,0 & 996 & 100,0 & & \\
\hline
\end{tabular}


Características de variáveis selecionadas das internações hospitalares realizadas pelo Sistema Único de Saúde em Belo Horizonte, Minas Gerais, Brasil, segundo hipótese diagnóstica e via de internação, 2002.

\begin{tabular}{|c|c|c|c|c|c|}
\hline \multirow[t]{2}{*}{ Variável } & \multicolumn{2}{|c|}{ Central de Internação } & \multicolumn{2}{|c|}{ Direta } & \multirow[t]{2}{*}{$p * \star$} \\
\hline & Mediana & Q1-Q3 * & Mediana & Q1-Q3 ** & \\
\hline \multicolumn{6}{|l|}{ Todas as internações } \\
\hline Número de dias em UTI & 3,0 & $2,0-3,0$ & 3,0 & $2,0-4,0$ & 0,081 \\
\hline Número de dias de internação & 6,5 & $4,0-10,0$ & 5,0 & $3,0-9,0$ & $<0,001$ \\
\hline \multicolumn{6}{|l|}{ Infarto agudo do miocárdio } \\
\hline Número de dias em UTI & 3,0 & $2,0-3,0$ & 3,0 & $2,0-4,0$ & 0,001 \\
\hline Número de dias de internação & 7,0 & $6,0-10,0$ & 7,0 & $5,0-12,5$ & 0,918 \\
\hline \multicolumn{6}{|l|}{ Insuficiência coronariana aguda } \\
\hline Número de dias em UTI & 3,0 & $2,0-4,0$ & 3,0 & $2,0-4,0$ & 0,964 \\
\hline Número de dias de internação & 6,0 & $4,0-9,0$ & 5,0 & $3,0-8,0$ & $<0,001$ \\
\hline
\end{tabular}

Internação mediou a internação hospitalar, é a característica da oferta.

Em 2001 a assistência médica hospitalar no SUS-BH estava apoiada em instituições privadas e filantrópicas, com carência de leitos de cardiologia no setor público e problemas com contratos/convênios com os prestadores hospitalares, inexistindo regras claras sobre a prestação de serviços. O sistema de informação era insuficiente para o controle sobre a utilização de leitos para internação pela Central de Internação ( $A$ Central de Internação no Processo de Regulação do SUS-BH; 2001. Documento de circulação interna da SMSA-BH). Segundo dados do Departamento de Informática do SUS - DATASUS (Santos RG, comunicação pessoal obtida por meio eletrônico na Gerência de Sistema de Infra-estrutura do SUS, DATASUS; 2005), em agosto de 2003 existiam 302 leitos de cardiologia em Belo Horizonte, dos quais 247 eram conveniados ao SUS. Dentre eles, apenas 3,6\% pertenciam aos hospitais públicos e estavam concentrados em dois serviços. Logo, o maior percentual de internações para os diagnósticos estudados era realizado em leitos da clínica médica. Por outro lado, existiam três hospitais públicos com porta aberta para atendimento ambulatorial de urgências, além das unidades ambulatoriais para atendimento às urgências e emergências, aumentando a demanda por internações, sem a devida retaguarda de leitos hospitalares. Somado a isso, um número crescente de serviços contratados vem garantindo sua sobrevivência financeira por intermédio de planos de saúde, filtrando pacientes que podem ou não utilizar determinados serviços, segundo critérios econômicos 15 , levando a um processo de retração progressiva da oferta de serviços para o SUS. Esses fatores explicam em parte a baixa oferta de leitos para internação, à ocasião, por tais procedimentos, pela Central de Internação. Por outro lado, existiam dificuldades com a regulação dos hospitais, que ocupavam seus leitos especialmente com pacientes oriundos de sua própria unidade de pronto atendimento e também uma provável priorização de internações de usuários do SUS por encaminhamento por profissionais de um serviço hospitalar a outro serviço.

Observou-se que nem todos os pacientes com hipótese diagnóstica de infarto agudo do miocárdio foram internados em UTI. Este é um recurso fundamental no cuidado desta doença, que interfere no resultado da assistência efetuada. Existiam em agosto de 2003, 251 leitos no município, sendo 127 conveniados ao SUS, dos quais, $51,2 \%$ pertenciam a hospitais públicos, segundo dados do DATASUS (Santos RG, comunicação pessoal obtida por meio eletrônico na Gerência de Sistema de Infra-estrutura do SUS, DATASUS; 2005). Com a escassez de leitos, para os pedidos de internação que chegavam à Central de Internação, havia a avaliação caso a caso pelos dados clínicos, exames informados e discussão direta com o médico que atendia ao caso, pelos médicos reguladores. Estes liberavam as vagas para a internação de acordo com a avaliação da gravidade do paciente, para serviços com complexidade compatível à necessidade assistencial. Como a seleção de casos foi realizada pela hipótese diagnóstica inicial e não por diagnóstico confirmado, certamente, além da dificuldade de obtenção de leitos para todos os casos, foram incluídos neste grupo diagnóstico pacientes com dor torácica aguda não coronariana. 
Apesar da pequena proporção de internações realizadas, os dados sugerem que a Central de Internação cumpriu parte de suas atribuições, viabilizando e priorizando internações de casos de maior gravidade, percebidos indiretamente pela maior proporção de internação de casos de infarto agudo do miocárdio e com utilização de UTI, quando comparada à via direta.

É necessária a realização de análises mais aprofundadas da estrutura e processo da assistência, comparando-se o desempenho dos serviços segundo as vias de internação. Devem ser levadas em consideração as características e a complexidade tanto dos serviços solicitantes quanto dos hospitais da internação, o perfil de gravidade dos pacientes e os recursos consumidos durante a hospitalização para se chegar a conclusões mais precisas com relação às diferenças nas características das internações das duas vias.

Destaca-se também no estudo a questão da proximidade geográfica como determinante do acesso e utilização de serviços. Acessibilidade aos serviços é a relação entre a localização da oferta e a localização dos usuários, levando-se em conta os recursos de transporte destes, o tempo, distância e os custos envolvidos no deslocamento ${ }^{3}$. As internações diretas apresentaram percentual mais significativo de internações de residentes em Belo Horizonte em comparação às internações pela Central de Internação, favorecidas provavelmente pela facilidade de acesso dos pacientes às unidades de atendimento às urgências e emergências em hospitais localizados nas proximidades de sua residência, ou por facilidades no seu deslocamento até tais áreas. A Central de Internação, por outro lado, mostrouse importante porta de entrada para as internações de residentes em outros municípios. As internações realizadas, segundo hipótese diagnóstica e via de acesso, quando estratificadas pela residência do paciente, mostraram maior proporção de internações por infarto agudo do miocárdio pela Central de Internação que por via direta, em residentes em outros municípios. O dado reforça a idéia do cumprimento, em parte, do papel da Central de Internação, ao viabilizar o acesso de população referenciada para assistência em serviços de maior complexidade em Belo Horizonte.
Vale ressaltar que não foram realizados todos os ajustes necessários, diante da indisponibilidade de variáveis clínicas que poderiam influenciar e contribuir para explicar as diferenças encontradas. Seria importante desenvolver estudos complementares que avaliem o tempo para atendimento do pedido de internação, os resultados obtidos, e os casos que não tiveram acesso às internações, embora tenha havido solicitação de vaga. Deve-se chamar atenção que no estudo foi utilizada base de dados de fonte criada para fins administrativos, fato que poderia afetar a qualidade de alguns dados utilizados no estudo. A SMSA-BH conta com uma equipe de médicos supervisores capacitados, que fiscalizam e verificam diariamente a pertinência das internações, o tipo de admissão, a assistência prestada, as informações das AIH com relação ao diagnóstico (procedimentos solicitados, realizados e cobrados) e à revisão de faturas hospitalares antes do encaminhamento ao nível central da SMSA-BH (Controle e Avaliação), minimizando este problema. Estudos realizados também apontaram melhoria na qualidade dos dados e confiabilidade alta para os procedimentos, como para outras variáveis referentes às internações e características dos pacientes 16,17,18,19.

As centrais de internação são ferramentas importantes para mediar o acesso aos serviços hospitalares, mormente em situações de urgência e emergência, otimizando recursos quando eles forem insuficientes para a demanda da população, sendo estratégicas para propiciar assistência mais equânime e de qualidade aos usuários do SUS. O acesso ótimo significa o fornecimento do cuidado adequado, no momento e local adequados 20. O estudo evidenciou que a gestão de leitos públicos e contratados pela Central de Internação foi insuficiente, mas há indícios de que a Central cumpre parcialmente seus objetivos, quando acionada. Diversas intervenções têm sido realizadas pela SMSA-BH, visando corrigir as distorções detectadas, e é possível que a situação tenha se modificado. Este estudo reforça a necessidade de realizar análises sistemáticas para identificar novos fatores que possam subsidiar mudanças, a fim de qualificar as Centrais de Internação cada vez mais para o exercício de seu papel, garantindo a eqüidade no acesso e qualidade na assistência prestada à população. 


\section{Resumo}

A Central de Internações de Belo Horizonte, Minas Gerais, Brasil, visa tornar ágil o acesso às internações pelo Sistema Único de Saúde (SUS). Entretanto, muitas internações ocorrem diretamente nos hospitais, sem intermediação da Central de Internação. O estudo comparou as características das internações realizadas em 2002, com relação à via de acesso. Foram selecionadas internações com hipótese diagnóstica de infarto agudo do miocárdio e insuficiência coronariana aguda. De 3.705 internações, 24,9\% foram realizadas pela Central de Internação e 75,1\% por via direta. As proporções de internações via direta foram maiores que pela Central de Internação para pacientes $\geq 70$ anos, internados por insuficiência coronariana aguda, na clínica cirúrgica e no fim de semana. Os percentuais das internações via Central de Internação foram maiores que os feitos por via direta para residentes em outros municípios, em hospitais não públicos e com utilização de UTI. O número de dias de internação também foi diferente entre as vias. O estudo mostrou diferenças nas características das internações realizadas pelas duas vias de acesso.

Doença Isquêmica do Coração; Acesso aos Serviços de Saúde; Hospitalizações

\section{Referências}

1. Ministério da Saúde. Lei Orgânica da Saúde no. 8.080. Diário Oficial da União 1990; 20 set.

2. Travassos C, Martins M. Uma revisão sobre os conceitos de acesso e utilização de serviços de saúde. Cad Saúde Pública 2004; 20 Suppl 2:S190-8.

3. Penchansky R, Thomas JW. The concept of access: definition and relationship to consumer satisfaction. Med Care 1981; 19:127-40.

4. Frenk J. El concepto y la medición de accesibilidad. Salud Pública Méx 1985; 27:438-53.

5. Donabedian A. The seven pillars of quality. Arch Pathol Lab Med 1990; 114:1115-8.

\section{Colaboradores}

P. A. Evangelista participou de todas as etapas do estudo, na elaboração, na revisão bibliográfica, análise dos dados e elaboração do artigo e sua redação final. S. M. Barreto e H. L. Guerra foram responsáveis pela orientação geral do estudo e participaram também de todas as etapas do trabalho, na elaboração do desenho metodológico do estudo, análise dos dados, discussão e revisão dos resultados, redação do artigo e revisão da redação final.
6. Departamento de Descentralização da Gestão da Assistência, Secretaria de Assistência à Saúde, Ministério da Saúde. Regionalização da assistência à saúde: aprofundando a descentralização com eqüidade no acesso. Norma Operacional da Assistência à Saúde - NOAS-SUS 01/02 (Portaria MS/ GM no. 373, de 27 de fevereiro de 2002, e regulamentação complementar). http://dtr2001.saude. gov.br/sas/caderno\%20NOAS\%2002.pdf (acessado em 08/Mar/2004). 
7. Unglert CVS. O enfoque da acessibilidade no planejamento da localização e dimensão de serviços de saúde. Rev Saúde Pública 1990; 24:445-52.

8. Franco SC, Campos GWS. Acesso a ambulatório pediátrico de um hospital universitário. Rev Saúde Pública 1998; 32:352-60.

9. Silva NN, Pedroso GC, Puccini RF, Furlani WJ. Desigualdades sociais e uso de serviços de saúde: evidências de análise estratificada. Rev Saúde Pública 2000; 34:44-9.

10. Assis MMA, Villa TCS, Nascimento MAA. Acesso aos serviços de saúde: uma possibilidade a ser construída na prática. Ciênc Saúde Coletiva 2003; 8:815-23.

11. Ramos DD, Lima MADS. Acesso e acolhimento aos usuários em uma unidade de saúde de Porto Alegre, Rio Grande do Sul, Brasil. Cad Saúde Pública 2003; 19:27-34.

12. Lopes RM, Vieira-da-Silva LM, Hartz ZMA. Teste de uma metodologia para avaliar a organização, acesso e qualidade técnica do cuidado na atenção à diarréia na infância. Cad Saúde Pública 2004; 20 Suppl 2:S283-97.

13. Oliveira EXG, Travassos C, Carvalho MS. Acesso à internação hospitalar nos municípios brasileiros em 2000: territórios do Sistema Único de Saúde. Cad Saúde Pública 2004; 20 Suppl 2:S298-309.
14. Organização Mundial da Saúde. Classificação estatística internacional de doenças e problemas relacionados à saúde, 10a revisão. v. 1. São Paulo: Centro Colaborador da OMS para a Classificação de Doenças em Português; 1995.

15. Cecilio LCO. Modelos tecno-assistenciais em saúde: da pirâmide ao círculo, uma possibilidade a ser explorada. Cad Saúde Pública 1997; 13:469-78.

16. Veras CMT, Martins MS. A confiabilidade dos dados nos formulários de Autorização de Internação Hospitalar (AIH), Rio de Janeiro, Brasil. Cad Saúde Pública 1994; 10:339-55.

17. Gouvea CSD, Travassos C, Fernandes C. Produção de serviços e qualidade da assistência hospitalar no Estado do Rio de Janeiro, Brasil - 1992 a 1995. Rev Saúde Pública 1997; 31:601-17.

18. Mathias TAF, Soboll MLMS. Confiabilidade de diagnósticos nos formulários de Autorização de Internação Hospitalar. Rev Saúde Pública 1998; 32:526-32.

19. Escosteguy CC, Portela MC, Medronho RA, Vasconcellos MTL. O Sistema de Informações Hospitalares e a assistência ao infarto agudo do miocárdio. Rev Saúde Pública 2002; 36:491-9.

20. Rogers A, Flowers J, Pencheon D. Improving access needs a whole systems approach. And will be important in averting crises in the millennium winter. BMJ 1999; 319:866-7.

Recebido em 30/Mar/2006

Versão final reapresentada em 22/Ago/2007 Aprovado em 20/Set/2007 